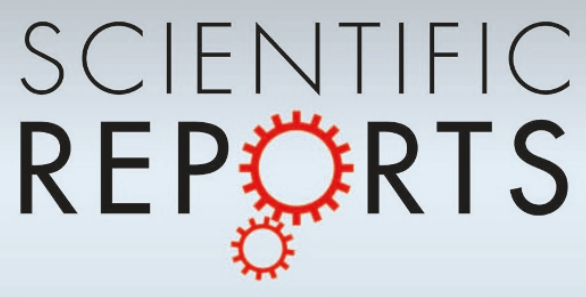

OPEN

SUBJECT AREAS:

COMPUTATIONAL MODELS

COMPUTATIONAL CHEMISTRY

Received

25 September 2014

Accepted

2 January 2015

Published

28 January 2015

Correspondence and requests for materials should be addressed to Y.T. lytang234@ecust. edu.cn) or Y.T. (tu@

theochem.kth.se)

\title{
Residues remote from the binding pocket control the antagonist selectivity towards the corticotropin-releasing factor
} receptor-1

\author{
Xianqiang Sun' ', Jianxin Cheng ${ }^{2}$, Xu Wang' , Yun Tang ${ }^{2}$, Hans Ågren' \& Yaoquan Tu' \\ 'Division of Theoretical Chemistry and Biology, School of Biotechnology, KTH Royal Institute of Technology, S-106 91 Stockholm, \\ Sweden, ${ }^{2}$ Shanghai Key Laboratory of New Drug Design, School of Pharmacy, East China University of Science and Technology, \\ Shanghai 200237, China.
}

The corticotropin releasing factors receptor- 1 and receptor- $2\left(C R F_{1} R\right.$ and $\left.C R F_{2} R\right)$ are therapeutic targets for treating neurological diseases. Antagonists targeting $C_{R F} R$ have been developed for the potential treatment of anxiety disorders and alcohol addiction. It has been found that antagonists targeting $C_{2} F_{1} R$ always show high selectivity, although $C R F_{1} R$ and $C R F_{2} R$ share a very high rate of sequence identity. This has inspired us to study the origin of the selectivity of the antagonists. We have therefore built a homology model for $C_{2 R} F_{2}$ and carried out unbiased molecular dynamics and well-tempered metadynamics simulations for systems with the antagonist $C P-376395$ in $C R F_{1} R$ or $C R F_{2} R$ to address this issue. We found that the side chain of $\mathrm{Tyr}^{6.63}$ forms a hydrogen bond with the residue remote from the binding pocket, which allows $\mathrm{Tyr}^{6.63}$ to adopt different conformations in the two receptors and results in the presence or absence of a bottleneck controlling the antagonist binding to or dissociation from the receptors. The rotameric switch of the side chain of Tyr356 $6^{6.63}$ allows the breaking down of the bottleneck and is a perquisite for the dissociation of CP-376395 from $\mathrm{CRF}_{1} \mathrm{R}$.

$\mathrm{T}$ he corticotropin-releasing factor $(\mathrm{CRF})$ receptor- $1\left(\mathrm{CRF}_{1} \mathrm{R}\right)$ and $\mathrm{CRF}$ receptor-2 $\left(\mathrm{CRF}_{2} \mathrm{R}\right)$ are family $\mathrm{B} \mathrm{G}$ protein-coupled receptors (GPCRs) composed of seven transmembrane helices (TM1 -TM7) linked by three intracellular loops (ICL1 -ICL3) and three extracellular loops (ECL1 -ECL3) ${ }^{1}$. CRF 1 R and $\mathrm{CRF}_{2} \mathrm{R}$ belong to the subfamily of CRF receptors and have been identified to be widely distributed throughout the central nervous system and periphery nervous system and act as key regulators of the hypothalamus-pituitary-adrenal axis ${ }^{2-4}$. It is believed that a well-balanced opposing action between $C^{2} F_{1} R$ and $C_{2 R F} R$ is responsible for the initiation of and the recovery from an elicited stress response and that a failed adaptation of the two receptors could lead to neuropathology, including anxiety and depression. Recent studies have revealed that $\mathrm{CRF}_{1} \mathrm{R}$ and $\mathrm{CRF}_{2} \mathrm{R}$ are involved in stress-associated anxiety and depression-like behavior in a more complicated way ${ }^{4-6}$. Selectively blocking of $\mathrm{CRF}_{1} \mathrm{R}$ or $\mathrm{CRF}_{2} \mathrm{R}$ with an antagonist is an effective way to treat the neuropathology. Efforts have been made to develop antagonists with high selectivity towards $\mathrm{CRF}_{1} \mathrm{R}$ or $\mathrm{CRF}_{2} \mathrm{R}$. Antagonists targeting $\mathrm{CRF}_{1} \mathrm{R}$ were among the first allosteric GPCR ligands to be evaluated clinically for treating depression and anxiety related disorders ${ }^{7}$.

In a GPCR subfamily, residues in the ligand binding pocket of the GPCRs are highly conserved, which can lead to the side effects posed by off-target effects ${ }^{8}$. It is interesting to note that sequence conservation in the subfamily of CRF receptors is even higher than in most of the other GPCR subfamilies. $C_{2 R} R$ and $C_{2} F_{2} R$ show very high sequence conservation on the helices TM5 and TM6 and the residues that directly interact with the antagonists are identical. However, the antagonist CP-376395 in the crystal structure of $\mathrm{CRF}_{1} \mathrm{R}$ shows a 1000 fold lower affinity towards $\mathrm{CRF}_{2} \mathrm{R}$ than towards $\mathrm{CRF}_{1} \mathrm{R}^{9}$. It has been shown that residues along the ligand binding/dissociation pathway of a target can affect the efficacy of a drug through influencing the binding kinetics of the drug towards its target ${ }^{10}$. Therefore, we assume that residues remote from the binding pocket play a role for the selectivity of the antagonist CP-376395.

To study the selectivity of the antagonist CP-376395 towards the receptors $\mathrm{CRF}_{1} \mathrm{R}$ and the role of the remote residues in the selectivity, we built a homology model of $\mathrm{CRF}_{2} \mathrm{R}$ with $\mathrm{CRF}_{1} \mathrm{R}$ as the template and carried out 
unbiased molecular dynamics simulations and well-tempered metadynamics simulations for both $\mathrm{CRF}_{1} \mathrm{R}$ and $\mathrm{CRF}_{2} \mathrm{R}$ with $\mathrm{CP}-376395$ binding to them. The dissociation of CP-376395 from $\mathrm{CRF}_{1} \mathrm{R}$ or $\mathrm{CRF}_{2} \mathrm{R}$ was observed in the well-temped metadynamics simulations. We found that the hydrogen bond between His $228^{3.40}$ and Tyr356 $6^{6.63}$ in $\mathrm{CRF}_{1} \mathrm{R}$, which is absent in $\mathrm{CRF}_{2} \mathrm{R}$, plays a pivotal role in controlling the difference of the binding of CP-376395 towards $C_{R F} R$ and $\mathrm{CRF}_{2} \mathrm{R}$ (Throughout this paper, the superscript on a residue represents the Wootten generic residue numbering ${ }^{11}$ ).

\section{Results and Discussion}

Homology modeling of $\mathrm{CRF}_{2} \mathrm{R}$. $\mathrm{CRF}_{1} \mathrm{R}$ and $\mathrm{CRF}_{2} \mathrm{R}$ belong to the same family and share a high sequence identity. The identity rate is $73 \%$ if only the transmembrane parts of the receptors are considered. The sequence alignment of $C_{2 R} F_{2}$ to $C_{R F} R$ is shown in Figure $S 1$. We can see that the most conserved residues match each other (Table $\mathrm{S} 1$ ). A Richardson plot of the modeled $\mathrm{CRF}_{2} \mathrm{R}$ structure indicates that $98 \%$ of the residues are located in the allowed regions, reflecting that the structure is geometrically reasonable (Figure S2) ${ }^{12}$. The root mean square deviation (RMSD) between the crystal structure of $\mathrm{CRF}_{1} \mathrm{R}$ and the modeled structure of $\mathrm{CRF}_{2} \mathrm{R}$ is $0.01 \AA$ (Figure 1).

Comparison of the structures of $C_{R F} R$ and $C_{1} F_{2} R$. The residues in the antagonist binding pocket of $\mathrm{CRF}_{1} \mathrm{R}$ are very similar to those in the corresponding pocket of $\mathrm{CRF}_{2} \mathrm{R}$, and, in particular, the residues in the first shell around CP-376395 are conserved (Figure 1). The trimethylmesitylene motif on CP-376395 forms a T-shaped $\pi-\pi$ stacking interaction with the residue Phe $313^{5.51}$ in $\mathrm{CRF}_{1} \mathrm{R}$ or with the residue Phe $280^{5.51}$ in $\mathrm{CRF}_{2} \mathrm{R}$. The nitrogen atom on the dimethylpyridine group of the antagonist forms a hydrogen bond with the side chain of the residue Asn $312^{5.50}$ in $\mathrm{CRF}_{1} \mathrm{R}$ (Figure 1b) or with the residue Asn279.50 in $\mathrm{CRF}_{2} \mathrm{R}$ (Figure 1c) ${ }^{1}$. It has been suggested that residues Phe $232^{3.44}$ and
Tyr356 $6^{6.63}$ in $\mathrm{CRF}_{1} \mathrm{R}$ work as a bottleneck for the binding of CP-376395 to the binding pocket (Figure 1b). Residues Phe199 $9^{3.44}$ and Tyr323 $33^{6.63}$ in $\mathrm{CRF}_{2} \mathrm{R}$ are located in the corresponding region of the bottleneck residues in $C_{1} F_{1} R$ to restrict the binding of $C P-376395$ to $C_{R F} R$ (Figure 1c). Although the residues directly interacting with the antagonist CP-376395 as described above are conserved in these two receptors, CP-376395 is highly selective towards $\mathrm{CRF}_{1} \mathrm{R}$, with the $\mathrm{K}_{i}$ values $12 \mathrm{nM}$ towards $\mathrm{CRF}_{1} \mathrm{R}$ and $>10000 \mathrm{nM}$ towards $\mathrm{CRF}_{2} \mathrm{R}^{9}$. Some residues along the suggested antagonist binding pathways are different (Figure S3). Such differences lead us to assume that the residues remote from the binding pocket likely affect the binding kinetics to control the selectivity of CP-376395 towards $\mathrm{CRF}_{1} \mathrm{R}$. Therefore, we performed unbiased molecular dynamics simulations and well-tempered metadynamics simulations to explain the differences.

MD simulations. As we can see from the RMSD curves in Figure 2a, $\mathrm{CRF}_{1} \mathrm{R}$ is rather stable with the RMSD value less than $2.0 \AA$ during the whole simulation. The RMSD value of $C_{2 R F_{2}} R$ is always larger than that of $\mathrm{CRF}_{1} \mathrm{R}$ but is still less than $2.5 \AA$ during the whole simulation. We attribute the slightly large RMSD value of $C_{2} F_{2} R$ to the relaxation of the modeled structure. To evaluate the flexibility of the residues in $C_{R F} R$ and $C_{2} F_{2} R$, the root mean square fluctuation (RMSF) values of the two receptors are calculated. The RMSF values indicate that in $\mathrm{CRF}_{1} \mathrm{R}$ and $\mathrm{CRF}_{2} \mathrm{R}, \mathrm{TM} 1-\mathrm{TM} 7$ are much more stable than the loops connecting these helices. The RMSF values of the two receptors calculated from the MD simulation trajectories were found to follow the same trend of those calculated from the B-factors of the $\mathrm{x}$-ray crystallography structure of $\mathrm{CRF}_{1} \mathrm{R}$ (Figure S4).

The kink of TM7. Helices TM1-TM7 form a bundle for the binding of the antagonist and for the signal transmission in the GPCRs. A
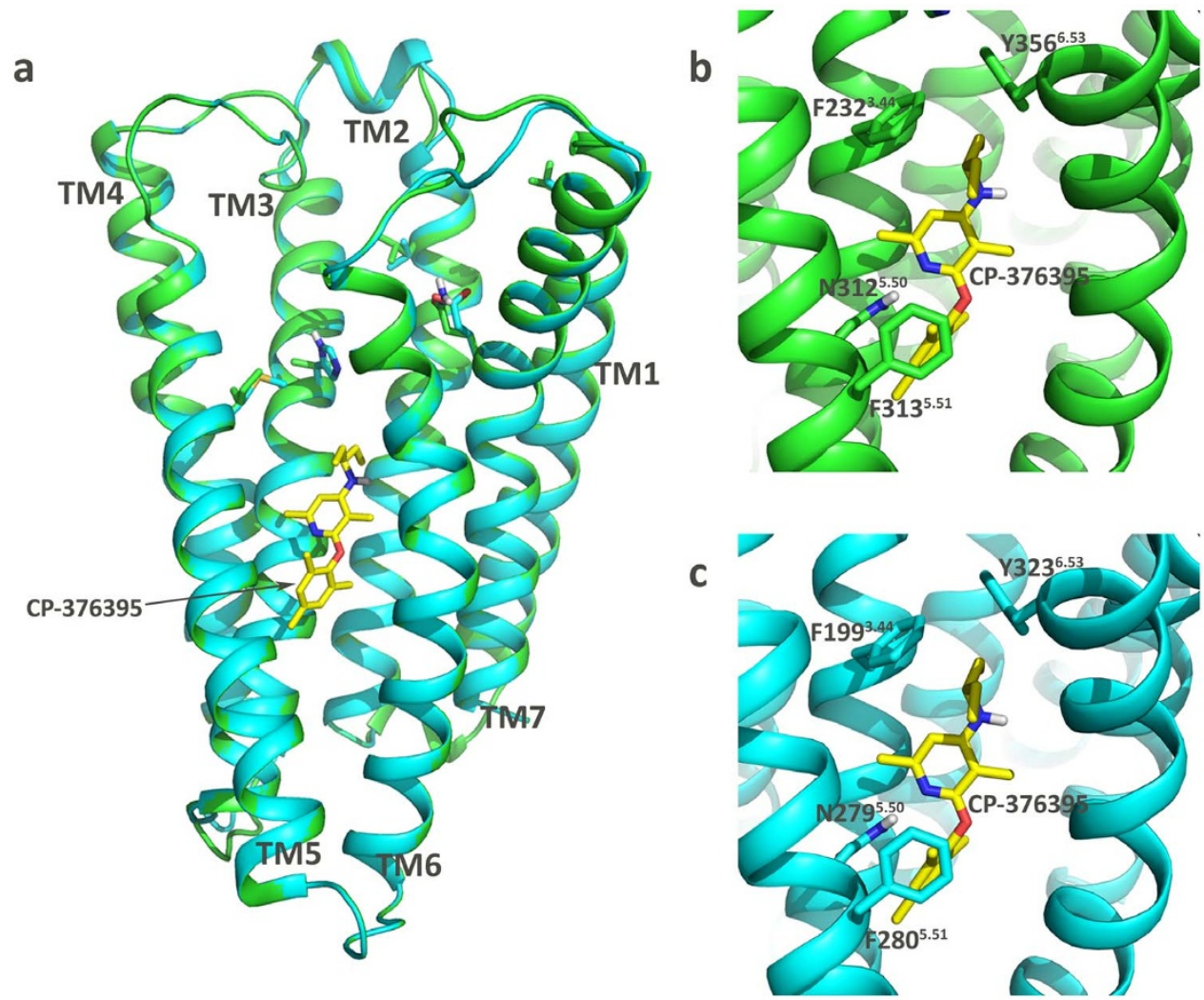

Figure $1 \mid$ The crystal structure of $\mathrm{CRF}_{1} \mathrm{R}$ and the modeled structure of $\mathrm{CRF}_{2} \mathrm{R}$. The protein structures are shown in the cartoon mode and the antagonist CP-376395 is shown in the stick mode. The structures of $\mathrm{CRF}_{1} \mathrm{R}$ and $\mathrm{CRF}_{2} \mathrm{R}$ are colored in green and cyan, respectively. The antagonist CP-376395 is colored in yellow. (a) Alignment of the crystal structure of $\mathrm{CRF}_{1} \mathrm{R}$ and the modeled structure of $\mathrm{CRF}_{2} \mathrm{R}$; (b) Key residues in the antagonist binding pocket of $C_{2 R} R$; (c) Key residues in the antagonist binding pocket of $C_{2 R F} R$. 

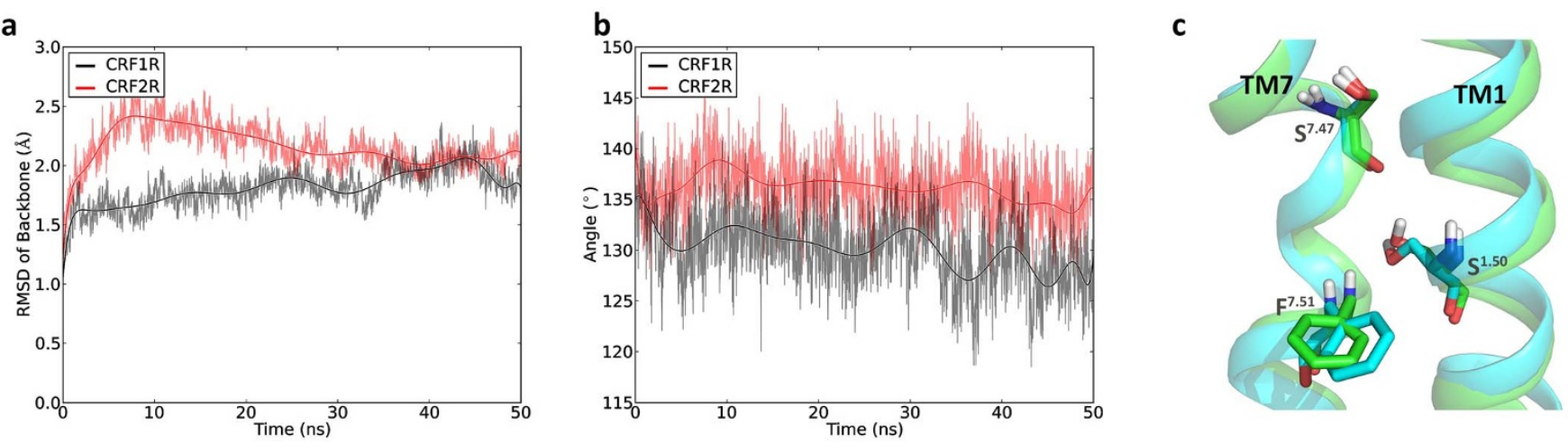

Figure 2 (a) RMSD values of the backbone atoms with respect to the first snapshots in the simulations; (b) Evolution of the tilt angle of TM7 with the pivot point at $\mathrm{Ser}^{7.47}$ in the simulations. (c) Structural representation of the key residues $\mathrm{Ser}^{1.50}, \mathrm{Ser}^{7.47}$ and Phe ${ }^{7.51}$ which control the kink of TM7 on $\mathrm{CRF}_{1} \mathrm{R}$ and $\mathrm{CRF}_{2} \mathrm{R}$. The structures of $\mathrm{CRF}_{1} \mathrm{R}$ and $\mathrm{CRF}_{2} \mathrm{R}$ are colored in green and cyan, respectively. The key residues are shown as sticks.

sharp kink of TM7 has been observed in the crystal structure of $\mathrm{CRF}_{1} \mathrm{R}^{1}$. We found that this kink is preserved during the MD simulation and that the kink angle is changing gradually from $135^{\circ}$ to $130^{\circ}$ with the pivot point at Ser382 $2^{7.47}$ (Figure $2 \mathrm{~b}$ and 2c). An analysis of the structure of $\mathrm{CRF}_{1} \mathrm{R}$ revealed that the kink is stabilized by the conserved residue Ser1301.50 which forms hydrogen bonds with the backbones of Ser382 $2^{7.47}$ and Phe $386^{7.51}$. The two hydrogen bonds were preserved during the $\mathrm{MD}$ simulation (Figure S5).

The structure of $\mathrm{CRF}_{2} \mathrm{R}$ was built based on the crystal structure of $\mathrm{CRF}_{1} \mathrm{R}$. Therefore, the sharp kink on TM7 and the two hydrogen bonds between the corresponding residues are kept in the modeled $\mathrm{CRF}_{2} \mathrm{R}$ structure (Figure $2 \mathrm{~b}$ and $2 \mathrm{c}$ ). The hydrogen bond between Ser127 $7^{1.50}$ and Phe $353^{7.51}$ is also preserved during the whole MD simulation, but the hydrogen bond between Ser127.50 and Ser349 ${ }^{7.47}$ broke in the beginning of the MD simulation (Figure S5). As a result, a smaller kink angle of TM7 was observed in $\mathrm{CRF}_{2} \mathrm{R}$ (Figure $2 \mathrm{~b}$ and $2 \mathrm{c}$ ). In family A GPCRs, such a kink has also been observed on TM7 with the residue $\operatorname{Pro}^{7.50}$ as the pivot point ${ }^{13,14}$.

The hydrogen bond between $\mathrm{His}^{2.50}$ and $\mathrm{Glu}^{3.50}$. Family B GPCRs lack the sequence motifs to form the conserved ionic lock connecting TM3 and TM6 as found in the inactive conformation of family A GPCRs'. In the family B GPCRs, a hydrogen bond between the residues $\mathrm{His}^{2.50}$ and $\mathrm{Glu}^{3.50}$ is believed to be involved in the receptor activation (Figure $3 \mathrm{a})^{15}$. In $\mathrm{CRF}_{1} \mathrm{R}$, the distance between $\mathrm{N}^{\delta}$ of His $184^{2.50}$ and $\mathrm{C}^{\delta}$ of Glu238 $38^{3.50}$ is about $4 \AA$ after $8 \mathrm{~ns}$ of the simulation and a hydrogen bond is formed between the side chains of His $184^{2.50}$ and Glu238 $38^{3.50}$ at the beginning of the simulation and is preserved in the simulation (Figure 3b). This is in line with the observation obtained by Bai. et $\mathrm{al}^{16}$. It can also be seen from Figure $3 \mathrm{~b}$ that the distances between $\mathrm{N}^{\delta}$ of His $184^{2.50}$ and $\mathrm{C}^{\delta}$ of Glu238 $8^{3.50}$ are predominantly around $4 \AA$. In contrast, the hydrogen bond between $\mathrm{His}^{2.50}$ and $\mathrm{Glu}^{3.50}$ is not stable in the simulation of $\mathrm{CRF}_{2} \mathrm{R}$. The $\mathrm{N}^{\delta}$ of His $152^{2.50}$ and $\mathrm{C}^{\delta}$ of Glu2053 $5^{3.50}$ became closer in about 5 ns to 7 ns but was separated thereafter with most of the distances distributed around $6 \AA$ as indicated in Figure $3 \mathrm{~b}$. The ionic lock between TM3 and TM6 in family A receptors is supposed to interconvert between the two states corresponding to the formation and breaking of the ionic $\operatorname{lock}^{17-20}$. Our simulation results also revealed that the potentially important hydrogen bond between $\mathrm{His}^{2.50}$ and $\mathrm{Glu}^{3.50}$ can interconvert between the formation and breaking of the hydrogen bond in family B GPCRs. Additionally, it is interesting for us to observe that in $\mathrm{CRF}_{2} \mathrm{R}$, Glu205 $5^{3.50}$ formed an ionic lock with $\operatorname{Arg} 148^{2.46}$ after 10 ns of the simulation and this ionic lock is preserved thereafter. The corresponding ionic lock in $\mathrm{CRF}_{1} \mathrm{R}$ was formed between Glu238 $38^{3.50}$ and $\operatorname{Arg} 180^{2.46}$ after $28 \mathrm{~ns}$ of the simulation and is preserved thereafter (Figure 3c). The free energy landscapes obtained from the metadynamics simulations clearly indicate that the locked state has a free energy of $5 \mathrm{kcal} / \mathrm{mol}$ lower than the unlocked state (Figure S6). This reflects that the system prefers to stay in the locked state. Thus, our results are in agreement with the observations from the long $\mathrm{MD}$ simulations ${ }^{18,21}$.

Ligand fluctuations in the unbiased MD simulations. CP-376395 binds to an unexpected site located in the cytoplasmic half of the receptor $\mathrm{CRF}_{1} \mathrm{R}$, which is about $18 \AA$ away from the putative agonist a

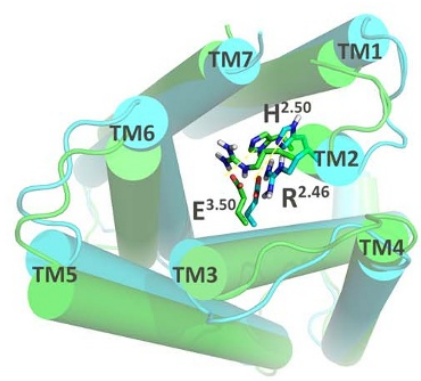

b

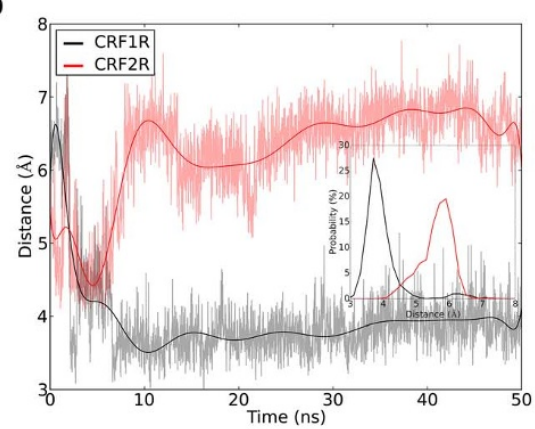

c

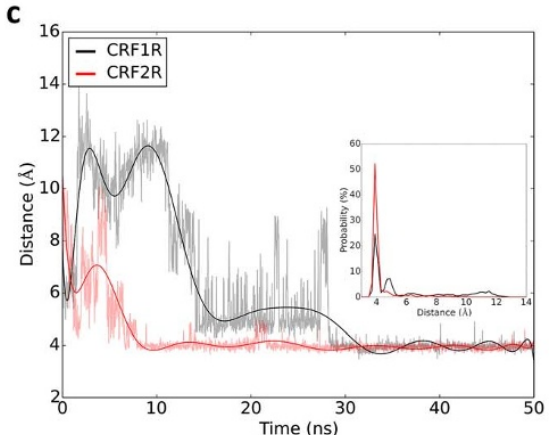

Figure $3 \mid$ (a) The final structures of the two receptors from the unbiased MD simulations viewed from the intracellular side. Residues His ${ }^{2.50}$, Arg ${ }^{2.46}$, and $\mathrm{Glu}^{3.50}$ are shown in the stick mode; (b) Evolution of the distances between $\mathrm{N}^{\delta}$ of $\mathrm{His}^{2.50}$ and $\mathrm{C}^{\delta}$ of $\mathrm{Glu}^{3.50}$ in the two receptors with the distributions shown in the middle panel; (c) Evolution of the distances between $\mathrm{C}^{\zeta}$ of $\mathrm{Arg}^{2.46}$ and $\mathrm{C}^{\delta}$ of $\mathrm{Glu}^{3.50}$ in the two receptors with the distributions shown in the middle panel. 
a

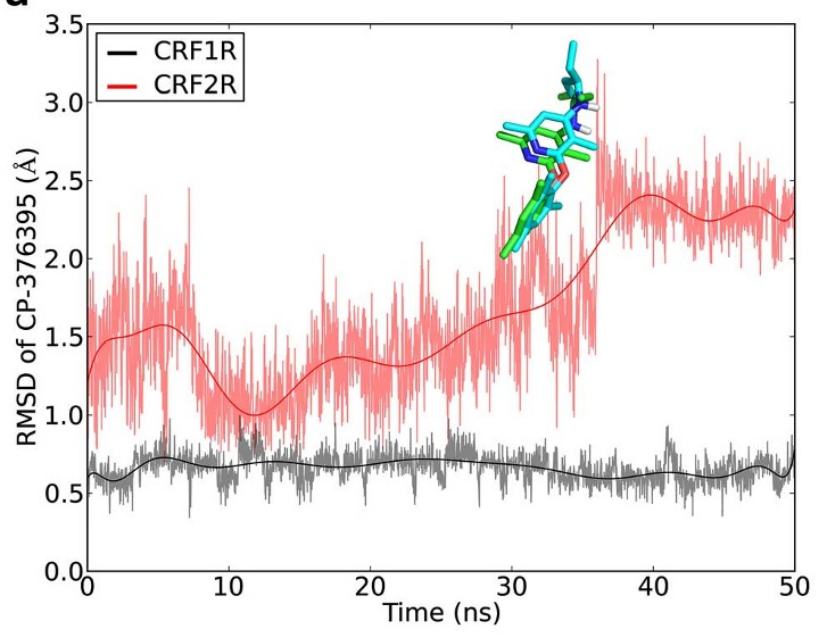

C

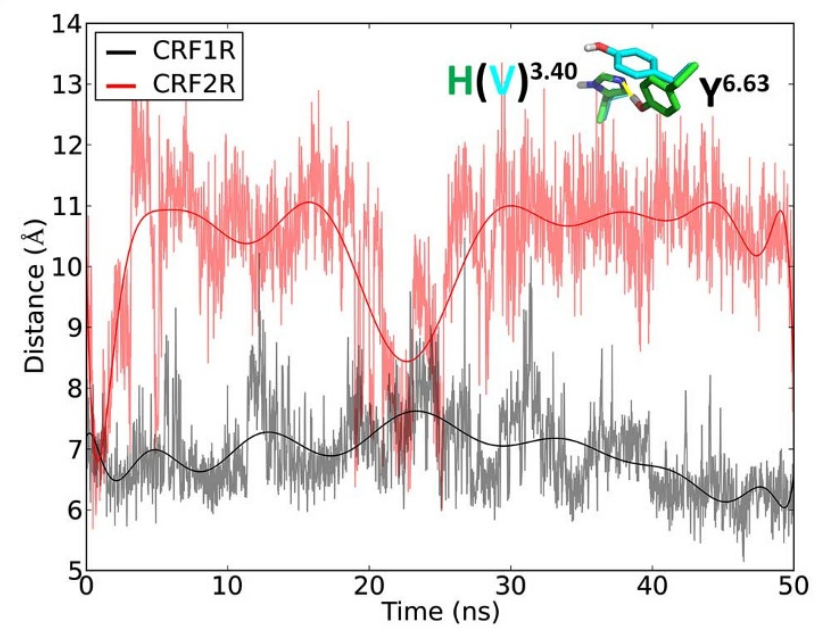

e

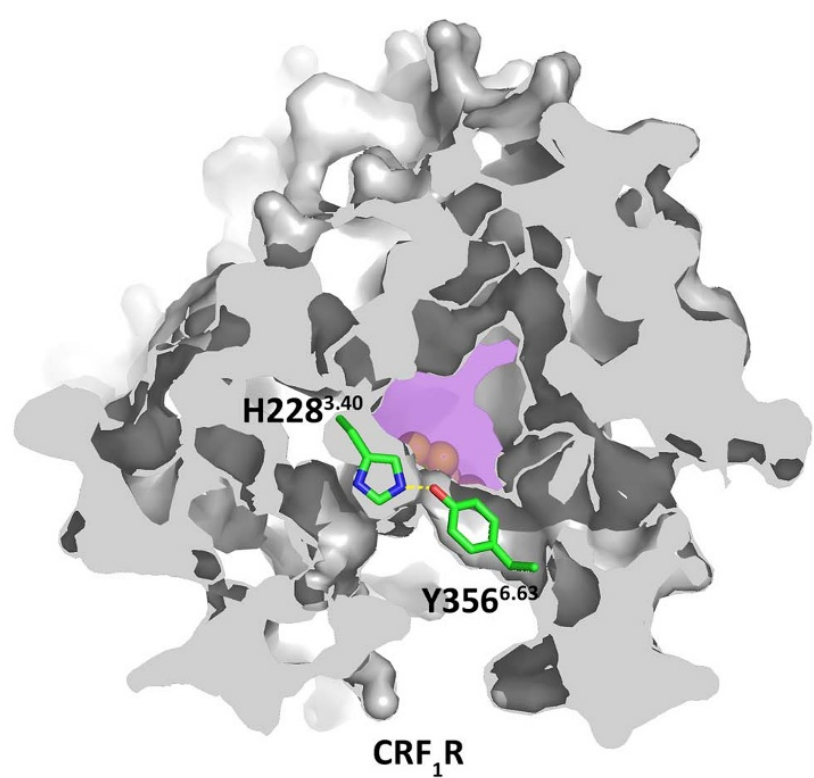

b

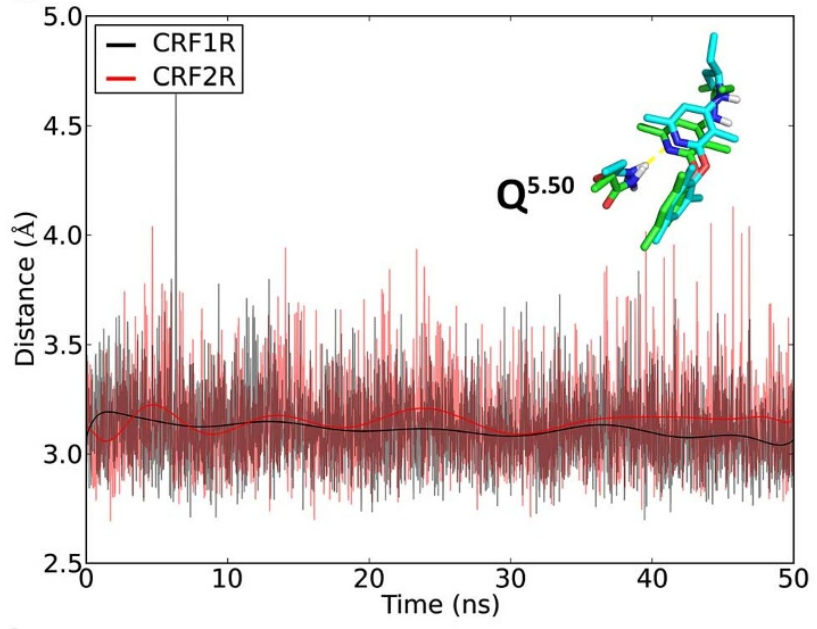

d

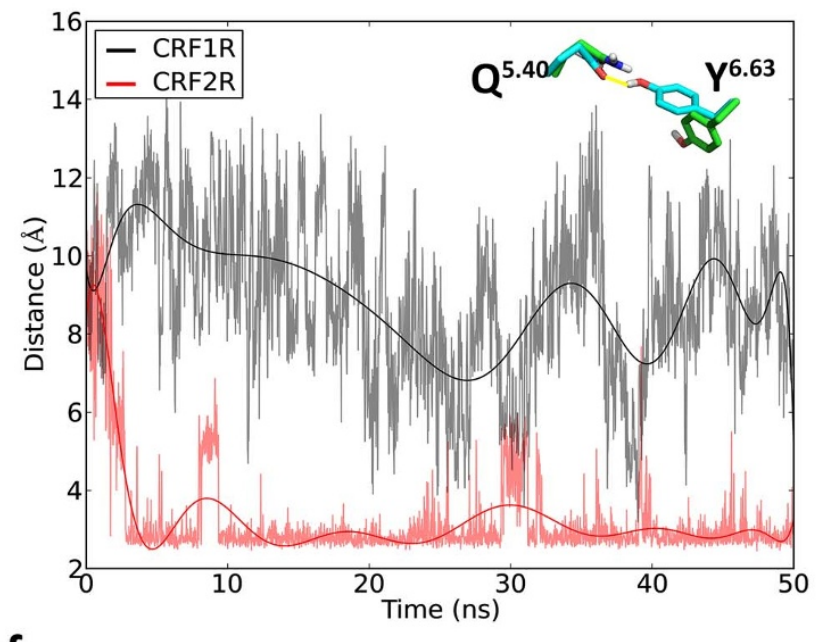

f

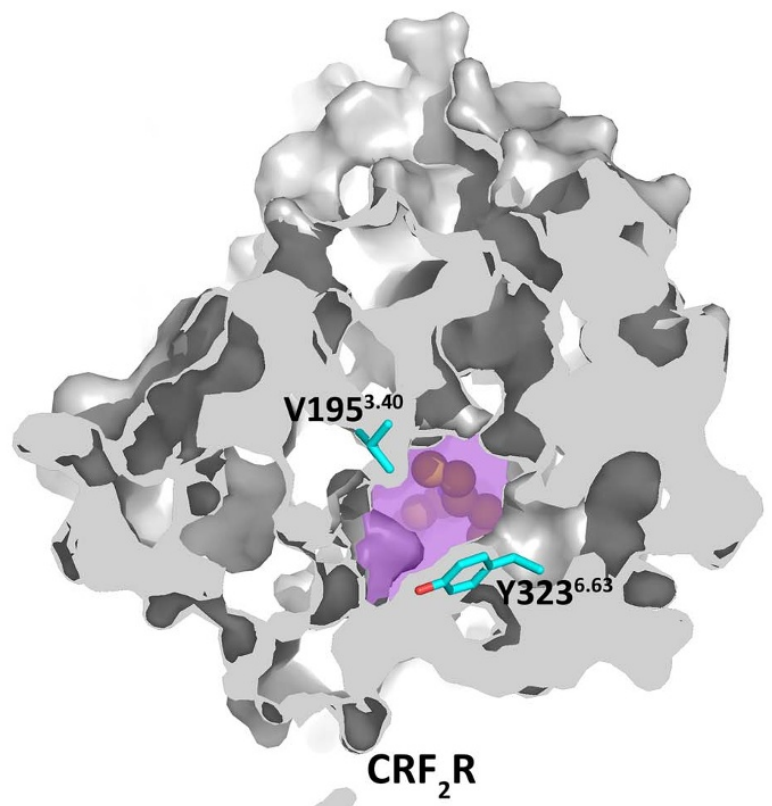

Figure $4 \mid$ (a) The RMSD values of the ligand with respect to the first snapshots of the simulations; (b) The distance between the side chain of Asn ${ }^{5.50}$ and the nitrogen on the pyridine ring of CP-376395; (c) The distance between the backbone carbon atoms on $\mathrm{Tyr}^{6.63}$ and $\mathrm{His}_{228^{3.40}}\left(\mathrm{CRF}_{1} \mathrm{R}\right)$ or between $^{6}$ the backbone carbon atoms on $\mathrm{Tyr}^{6.63}$ and Val228 ${ }^{3.40}\left(\mathrm{CRF}_{2} \mathrm{R}\right)$; (d) The distance between the side chain oxygen atoms on Tyr ${ }^{6.63}$ and $\mathrm{Gln}^{5.50}$; (e) Crosssection view of $\mathrm{CRF}_{1} \mathrm{R}$ with the bottleneck formed; ( $\mathrm{f}$ ) Cross-section view of $\mathrm{CRF}_{2} \mathrm{R}$ without the bottleneck. In (e) and (f), the antagonist binding pockets are colored in magenta. 
binding site of the receptor and is about $13-23 \AA$ away from the corresponding small ligand binding site of family A GPCRs ${ }^{16}$. Consistent with the observation of Bai et al., small RMSD values $(\sim 0.5 \AA)$ of the ligand were obtained from our simulation of $\mathrm{CRF}_{1} \mathrm{R}$. In contrast, CP-376395 shows RMSD values in the binding pocket of $C F_{2} R$ larger than in that of $C F_{1} R$ as indicated in Figure $4 \mathrm{a}$. The conserved residue $\mathrm{Asn}^{5.50}$ forms an essential hydrogen bond with the nitrogen on the pyridine ring of CP376395 and mutation of this residue to Ala results in a complete loss of ligand binding ${ }^{1}$. This key hydrogen bond is preserved during the simulations of both $\mathrm{CRF}_{1} \mathrm{R}$ and $\mathrm{CRF}_{2} \mathrm{R}$ and stabilizes the aryloxy moiety of CP-376395 (Figure 4b). Thus, the larger RMSD value of CP-376395 in $\mathrm{CRF}_{2} \mathrm{R}$ is mainly contributed by the fluctuation of the exocyclic alkylamino group.

The exocyclic alkylamino group is located adjacent to the residues $\mathrm{Phe}^{3.44}$ and $\mathrm{Tyr}^{6.63}$ of $\mathrm{CRF}_{1} \mathrm{R}$ (Figure $1 \mathrm{~b}$ ) and $\mathrm{CRF}_{2} \mathrm{R}$ (Figure 1c), which have been suggested to be the bottleneck for the binding of the antagonist to $\mathrm{CRF}_{1} \mathrm{R}^{1}$. The residue Tyr356 ${ }^{6.63}$ is located within the hydrogen bond distance of His $228^{3.40}$ in $\mathrm{CRF}_{1} \mathrm{R}$. However, the residue Val $195^{3.40}$ of $\mathrm{CRF}_{2} \mathrm{R}$ is corresponding to His $228^{3.40}$ of $\mathrm{CRF}_{1} \mathrm{R}$ and lacks the hydrogen bond donor or acceptor atoms on its side chain to form a hydrogen bond with Tyr323 $3^{6.63}$. In our simulations, the distance between the side chain oxygen atom of Tyr $356^{6.63}$ and $C^{\alpha}$ of His $228^{3.40}$ of $\mathrm{CRF}_{1} \mathrm{R}$ was preserved during the MD simulation, reflecting that the hydrogen bond formed between the side chains of Tyr356 $6^{6.63}$ and His $228^{3.40}$ was stable (Figure $4 \mathrm{~d}$ ). In contrast, the distance between the side chain of Tyr323 $3^{6.63}$ and $C^{\alpha}$ of Val195 $5^{3.40}$ in $\mathrm{CRF}_{2} \mathrm{R}$ became much larger in the first couple of nanoseconds and remained unchanged in the following simulation with a restoration of the initial value between $20 \mathrm{~ns}$ and $25 \mathrm{~ns}$. The increase of the distance alters the conformation of the side chain of Tyr323.63 in $\mathrm{CRF}_{2} \mathrm{R}$ (Figure $4 \mathrm{~d}$ and Figure S7) and this conformational change is not taking place randomly (Figure S8). Interestingly, the rotameric change of this residue generates a hydrogen bond between the side chains of Tyr323 $3^{6.63}$ and Gln $269^{6.44}$. This hydrogen bond was pre- served until the end of the $50 \mathrm{~ns}$ MD simulation to stabilize the rotameric change of Tyr323 $3^{6.63}$ (Figure $4 \mathrm{c}$ and Figure S7). Such rotameric switch results in the breaking down of the bottleneck (Figure 4f), while such a bottleneck is preserved during the simulation of $\mathrm{CRF}_{1} \mathrm{R}$ (Figure 4e). We thus assume that such a difference controls the CP-376395 to dissociate from the antagonist binding pocket in $\mathrm{CRF}_{1} \mathrm{R}$ and $\mathrm{CRF}_{2} \mathrm{R}$.

Metadynamics simulations. In our well-tempered metadynamics simulations, CP-376395 left the antagonist binding pocket in $\mathrm{CRF}_{1} \mathrm{R}$ and $\mathrm{CRF}_{2} \mathrm{R}$, and explored the binding pathways to exit the receptors through the helices bundle (Supporting videos). The free energy surfaces (FESs) for CP-376395 leaving the antagonist binding pockets of $C R F_{1} R$ and $C R F_{2} R$ are displayed in Figure 5 and Figure 6, respectively.

Metastable poses of CP-376395 in CRF $_{1} \mathbf{R}$. For CP-376395 in $\mathrm{CRF}_{1} \mathrm{R}$, we observed three energy minima (basin $\mathrm{B} 0$, basin $\mathrm{B} 1$, and basin B2) as displayed in the FES (Figure 5a). The deepest free energy minimum in the FES is depicted as basin B0 in Figure $5 \mathrm{~b}$ and corresponds to the conformation that CP-376395 adopts in the $\mathrm{x}$-ray crystallography structure. This conformation corresponds to the energetically most favorable pose for the binding of CP-376395 in the antagonist binding pocket of $\mathrm{CRF}_{1} \mathrm{R}$. The aryloxy moiety of $\mathrm{CP}$ 376395 is holding tightly in the binding pocket by strongly hydrogenbonding to Asn $312^{5.50}$ and by hydrophobic interactions with Leu316 $6^{5.54}$, Ile319 $9^{5.57}$, Thr345 $5^{6.42}$, Leu348 $8^{6.45}$, and Leu349. The exocyclic alkylamino group keeps interacting with Gly $353^{6.50}$, Phe232 $2^{3.44}$, Leu309 $9^{5.47}$, and Tyr356 $6^{6.63}$. The mesityl group on CP376395 forms a T-shaped $\pi-\pi$ stacking interaction with Phe $313^{5.51}$.

In the metadynamics simulation, CP-376395 moved along the helix bundle to the extracellular side half of the receptor $C_{R F} R$ and reached a position sufficiently close for interacting directly with the bottleneck residues Tyr356 6.63 and Phe232 3.44 (Figure 5c). The mesityl group of CP-376395 is placed in a hydrophobic cavity

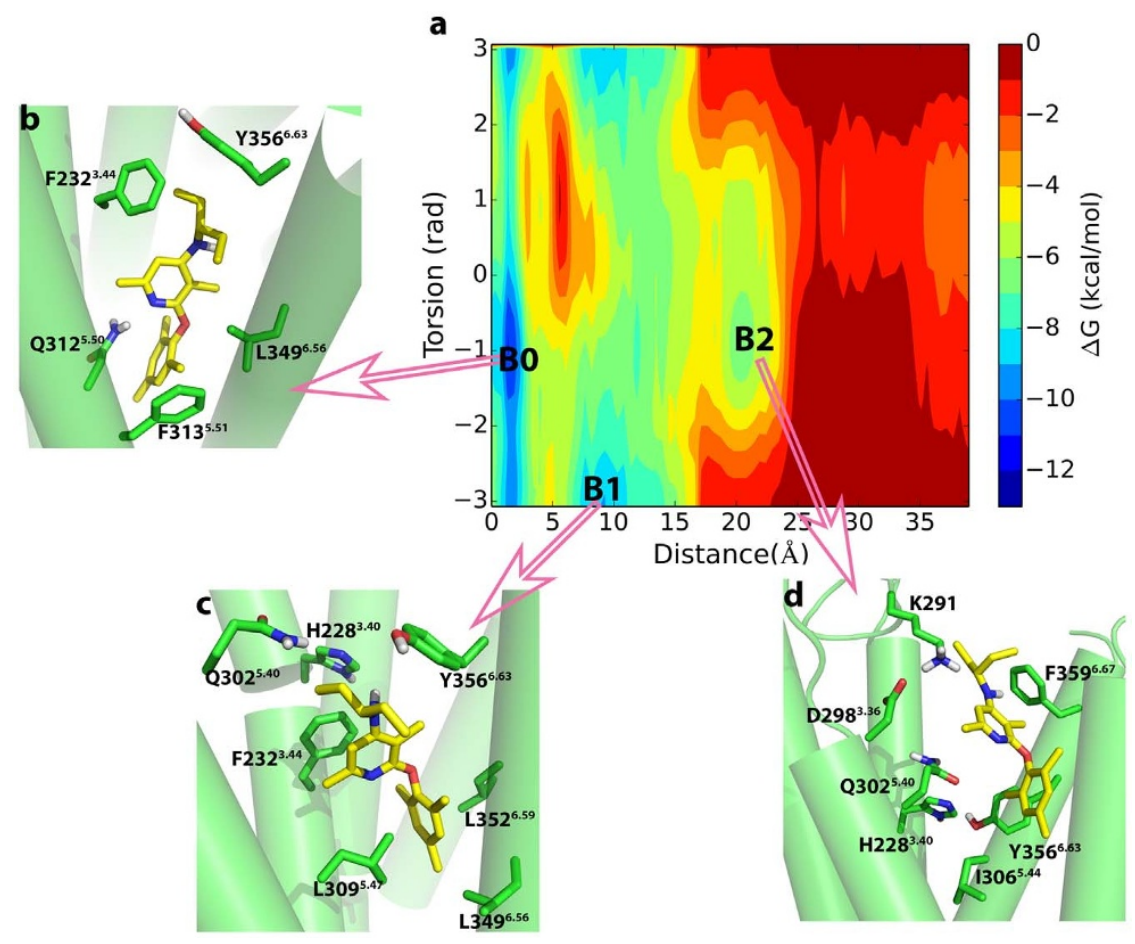

Figure 5 Metastable states in the dissociation of CP-376395 from $\mathrm{CRF}_{1} \mathrm{R}$. (a) The binding free energy surface for the dissociation of CP-376395 from $\mathrm{CRF}_{1} \mathrm{R}$ as a function of the $\mathrm{Z}$-component of the vector connecting the nitrogen on the dimethylpyridine group of CP-376395 and $\mathrm{C}^{\mathrm{Y}}$ on the Asn $312^{5.50}$ and $\chi_{1}$ torsional angle of Tyr356 $6^{6.63}$. The three main energy basins B0-B2 found in the metadynamics simulation are highlighted in b-d, respectively; (b)-(d) Structural characterization of the metastable states B0-B2. 
surrounded by Leu3095.47, Leu348 $8^{6.55}$, Leu349 $9^{6.56}$, Leu352 $2^{6.59}$, and Gly $353^{6.60}$. The dimethylpyridine group of the antagonist is surrounded by the residues Ile306 $6^{5.44}$, Gln302 $2^{5.40}$, and Tyr356 $6^{6.63}$. Met305 $5^{5.43}$ also interacts with the dimethylpyridine group. The pyridine ring of CP-376395 forms a face to face $\pi-\pi$ stacking interaction with the benzene ring of Phe $232^{3.44}$. This $\pi-\pi$ stacking interaction can stabilize the ligand in the binding site corresponding to the basin B1. In this basin, the torsional angle $\chi_{1}$ of the residue Tyr356 $6^{6.63}$ is around $\pi$ (or $-\pi$ ), while $\chi_{1}$ of Tyr356 $6^{6.63}$ is about -1 in basin B0. Such a difference indicates that a rotameric change of Tyr $356^{6.63}$ is required for CP-376395 moving from the site corresponding to the basin B0 to that corresponding to the basin B1.

Once all the metastable states in the helix bundle were filled, the antagonist CP-376395 crossed the helix bundle to reach the extracellular vestibule of $\mathrm{CRF}_{1} \mathrm{R}$ to form a metastable vestibule-bound state, where a local minimum (basin B2) was found as displayed in Figure $5 \mathrm{~d}$. The vestibule-bound state is the first step for CP-376395 to enter the antagonist binding site. In this local minimum, the antagonist CP-376395 is stabilized by the hydrophobic interactions with the residues Ile $306^{5.44}$, Phe $360^{6.67}$, and Tyr356 6.63 , and an additional amount of interaction energy can be gained from the relative closeness of the residues Asp298 $8^{3.36}$ and Lys291.

Metastable poses of CP-376395 in $\mathrm{CRF}_{2} \mathrm{R}$. We observe three energy minima (basin B0, basin B1 and basin B2) during the dissociation of the antagonist CP-376395 from $\mathrm{CRF}_{2} \mathrm{R}$ as displayed in Figure 6a. Basin B0 represents the energetically most stable site. This basin approximately corresponds to the crystallized conformation of CP376395 in $\mathrm{CRF}_{1} \mathrm{R}$ (Figure 6b). At the basin, the hydrophobic interactions between $\mathrm{CRF}_{2} \mathrm{R}$ and $\mathrm{CP}-376395$, which have been observed in the unbiased MD simulation, are kept. The dimethylpyridine group adopts a conformation with the nitrogen atom on the ring hydrogen-bonded to Asn2795.50, which allows
CP-376395 to hold tightly with the antagonist binding pocket. The hydrophobic interactions originating from Leu283.54, Ile286 ${ }^{5.57}$, Leu315 5.55 , and Leu316 5.56 further stabilize CP-376395 in this region. The exocyclic alkylamino group keeps interacting with Gly313 ${ }^{6.50}$, Phe199 $9^{3.44}$, Leu276 $6^{5.47}$, and Tyr323 ${ }^{6.63}$.

With the action of metadynamics, the antagonist CP-376395 moved along the helix bundle and reached another minimum displayed as basin B1 (Figure 6c). The traverse from basin B0 to basin B1 results in a breaking of the hydrogen bond between the nitrogen on the dimethylpyridine group of CP-376395 and the side chain of Asn $279^{5.50}$. This hydrogen bond was found to be preserved during our $50 \mathrm{~ns}$ of the unbiased MD simulation, reflecting the inability of the unbiased MD in traversing energy barriers to sample the conformation states at different energy minima. Here, the dimethylpyridine group of CP-376395 resides in the pocket surrounded by Phe $191^{3.36}$, Val195 $5^{3.40}$, Ile $321^{6.61}$, and Met $324^{6.64}$. The mesityl moiety on CP-376395 is in a highly hydrophobic cage defined by Leu316 ${ }^{5.56}$, Leu319 $9^{6.59}$, Phe199 $9^{3.44}$, and Leu276 $6^{5.47}$. It is interesting to observe the $\pi-\pi$ interaction between Tyr $323^{6.63}$ and the pyridine ring on CP376395 to stabilize the pose of CP-376395 in $\mathrm{CRF}_{2} \mathrm{R}$.

We observed another metastable state (basin B2) residing at about $10 \AA$ away from the residue Asn279.50 (Figure 6d). As shown in Figure 6a, a large region surrounding basins B1 and B2 is energetically favorable, with the torsional angle ranging from -1 to $-\pi$ and the distance kept at about $10 \AA$. This means that the torsional angle can change between 1 and $-\pi$ with a very low energy barrier. Interestingly, the $\pi-\pi$ interactions between Tyr $323^{6.63}$ and the pyridine ring on CP-376395 are kept in this energetically favorable region. On the other hand, the hydrogen bond between Tyr323 $3^{6.63}$ and Asn $269^{5.40}$ is broken in basins B1 and B2. We thus suggest that the $\pi-\pi$ interactions between Tyr323 $3^{6.63}$ and the pyridine ring on CP376395 compensate for the energy required for the breaking of the hydrogen bond between Tyr323 $3^{6.63}$ and Asn269.40.

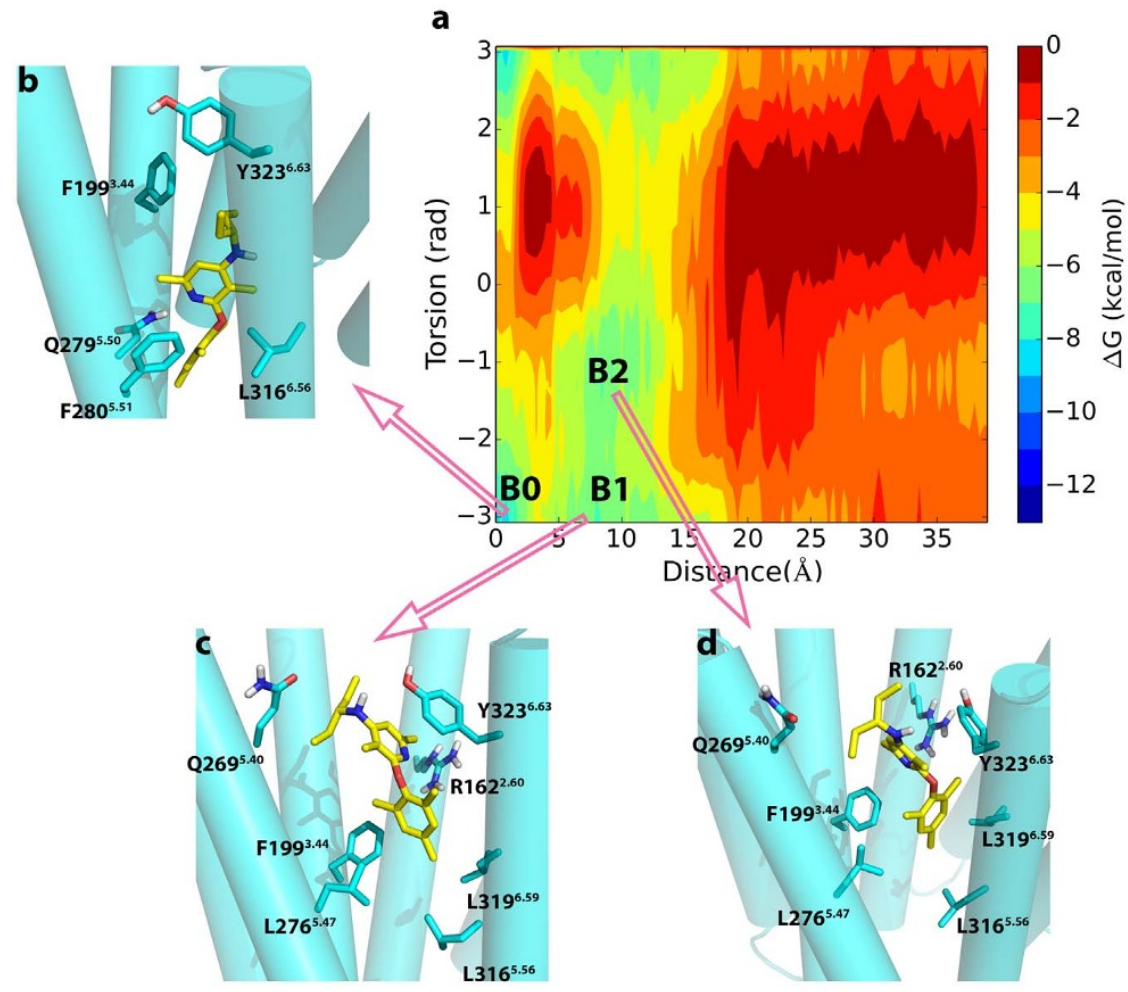

Figure 6 Metastable states in the dissociation of CP-376395 from $\mathrm{CRF}_{2} \mathrm{R}$. (a) The binding free energy surface for the dissociation of CP-376395 from $\mathrm{CRF}_{2} \mathrm{R}$ as a function of the Z-component of the vector connecting the nitrogen on the dimethylpyridine group of CP-376395 and $\mathrm{C}^{\gamma}$ on the Asn2795.50 and $\chi_{1}$ torsional angle of Tyr323 $3^{6.63}$. The three main energy basins B0-B2 found in the metadynamics simulation are highlighted in b-d, respectively; (b)-(d), Structural characterization of the metastable states B0-B2. 
Comparison of the ligand dissociation pathways and energy profiles. Three energy minima were identified for the dissociation of CP-376395 from both $\mathrm{CRF}_{1} \mathrm{R}$ and $\mathrm{CRF}_{2} \mathrm{R}$ as displayed in Figure 5 and Figure 6, respectively. As depicted in the figures, the basin $\mathrm{B} 0$ corresponds to the region located about 1-2 $\AA$ away from the key residue Asn ${ }^{5.50}$ with the torsional angle $\chi_{1}$ of the residue $\mathrm{Tyr}^{6.63}$ stabilized at -1 in $\mathrm{CRF}_{1} \mathrm{R}$ or $-\pi$ (or $\pi$ ) in $\mathrm{CRF}_{2} \mathrm{R}$. With CP-376395 moving from the basin $\mathrm{B} 0$ to the basin $\mathrm{B} 1$, the torsional angle $\chi_{1}$ of the residue Tyr356 $6^{6.63}$ in $\mathrm{CRF}_{1} \mathrm{R}$ switches from -1 to $-\pi$ (or $\pi$ ) while the corresponding angle in $\mathrm{CRF}_{2} \mathrm{R}$ stays at -1 (Figure S). Additionally, $\mathrm{Phe}^{3.44}$ displays no significant conformational change in neither the unbiased $\mathrm{MD}$ simulations nor in the metadynamics $\mathrm{MD}$ simulations of $\mathrm{CRF}_{1} \mathrm{R}$ or $\mathrm{CRF}_{2} \mathrm{R}$. These observations strongly support our suggestion that the conformational change of Tyr356 36.63 plays a pivotal role for $\mathrm{CP}$ 376395 binding to or dissociation from the antagonist binding site of $\mathrm{CRF}_{1} \mathrm{R}$. With the formation of the hydrogen bond between the side chains of the residues Tyr $323^{6.63}$ and $\mathrm{Gln} 269^{6.44}$ in $\mathrm{CRF}_{2} \mathrm{R}$, the crucial role of $\mathrm{Tyr}^{6.63}$ which controls the antagonist binding to or to dissociation from the binding site in $\mathrm{CRF}_{1} \mathrm{R}$ does not occur in $\mathrm{CRF}_{2} \mathrm{R}$.

Bai et al. explored the dissociation pathway of CP-376395 from $\mathrm{CRF}_{1} \mathrm{R}$ by using random acceleration molecular dynamics simulations ${ }^{16}$. They found that breaking of the hydrogen bond between CP-376395 and Asn $312^{5.50}$ results in the first energy barrier for the dissociation. This observation was confirmed from our study. Besides, we found that breaking of the hydrogen bond formed between Tyr356 $6^{6.63}$ and His $228^{3.40}$ also contributes to this barrier. The barrier is about $5 \mathrm{kcal} / \mathrm{mol}$ from our simulation, while it is $9.9 \mathrm{kcal} / \mathrm{mol}$ from Bai's work. Bai and coworkers also found the second energy barrier for the dissociation of CP-376395 along the pathway they detected because there exist two hydrogen bonds formed by CP-376395 with His $228^{3.40}$ and Gln302 $2^{5.40}$ in the pathway. However, these two hydrogen bonds were not observed along the CP-376395 dissociation pathway in our study. We found that CP376395 forms $\pi-\pi$ stacking interactions with Tyr356 $6^{6.63}$, which was not described by Bai. et al. The $\pi-\pi$ stacking interactions, together with the interactions between CP-376395 and the remaining residues, form the second energy barrier of about $4 \mathrm{kcal} / \mathrm{mol}$. This barrier is much lower than the one obtained by Bai et al., which is $11.4 \mathrm{kcal} / \mathrm{mol}$.

Implications for the drug design. The binding free energy for $\mathrm{CP}$ 376395 towards $\mathrm{CRF}_{1} \mathrm{R}$, averaged over those from the first and repeated runs $(-11.42$ and $-11.27 \mathrm{kcal} / \mathrm{mol}$, respectively), is $-11.35 \mathrm{kcal} / \mathrm{mol}$, while that for $\mathrm{CP}-376395$ towards $\mathrm{CRF}_{2} \mathrm{R}$, averaged over those from the two simulations $(-8.18 \mathrm{kcal} / \mathrm{mol}$ and $-8.03 \mathrm{kcal} / \mathrm{mol}$, respectively) is $-8.10 \mathrm{kcal} / \mathrm{mol}$ (Figures $\mathrm{S} 9$ and S10). The binding free energy for the CP-376395 towards $\mathrm{CRF}_{1} \mathrm{R}$ is in agreement with the experimental value $(-10.87 \mathrm{kcal} /$ mol) while that for the CP-376395 towards $\mathrm{CRF}_{2} \mathrm{R}$ is a little larger than the experimental result (weaker than $-6.86 \mathrm{kcal} / \mathrm{mol}$ ) ${ }^{9}$. The decrease of the antagonist binding affinity from $C_{2 R F_{1}} R$ to $C_{R F} R$ is in agreement with the experimental results.

Mutation of His $228^{3.40}$ has also been performed to evaluate the effect of His $228^{3.40}$ on the binding of the antagonist NBI27914, which shares a similar scaffold to CP-376395, towards $\mathrm{CRF}_{1} \mathrm{R}^{22}$. Compared to the 1000-fold higher binding affinity of CP-376395 towards $\mathrm{CRF}_{1} \mathrm{R}$ than towards $\mathrm{CRF}_{2} \mathrm{R}$, the His $288 \mathrm{Val}$ mutation only leads to a 40 -fold lower binding affinity of NBI27914 towards $\mathrm{CRF}_{1} \mathrm{R}$. Such a difference likely comes from two aspects. One aspect is that the exocyclic alkylamino group in NBI27914 is bigger than that in CP376395 , which results in the hydrogen bond between His $228^{3.40}$ and Tyr356 $6^{6.63}$ being less stable in the binding of NBI27914 to $\mathrm{CRF}_{1} \mathrm{R}$. Another aspect is that the decrease of the binding affinity of CP376395 to $\mathrm{CRF}_{2} \mathrm{R}$ is likely contributed partially by the difference in the rotameric properties of $\mathrm{Tyr}^{6.63}$ in $\mathrm{CRF}_{1} \mathrm{R}$ and $\mathrm{CRF}_{2} \mathrm{R}$.

Recent studies have indicated that the binding kinetics of a ligand towards its target could be one of the most crucial factors for sustainable drug efficacy, and in some cases, even more important than the binding affinity in determining the drug efficacy ${ }^{23,24}$. The recently published crystal structure of the smoothened receptor by Stevens's group revealed that there are multiple distinct binding sites for the ligand in the helix bundle of the receptor ${ }^{25,26}$. Both smoothened receptor and CRFR family receptors possess deep ligand binding cavities. This allows us to suggest that the sites along the pathway of an antagonist binding to the CRFR family of receptors, especially those corresponding the basins and saddle points we discovered in our metadynamics simulations, are important in the design of new drug candidates with attenuated side effects and chemoresistance.

\section{Conclusion}

In this work, we have carried out homology modeling to build the structure of $\mathrm{CRF}_{2} \mathrm{R}$ with the crystal structure of $\mathrm{CRF}_{1} \mathrm{R}$ as the template. Based on the crystal structure of $\mathrm{CRF}_{1} \mathrm{R}$ and the homology model of $\mathrm{CRF}_{2} \mathrm{R}$, we performed unbiased $\mathrm{MD}$ simulations as well as well-tempered metadynamics simulations to investigate the origin of the selectivity of the antagonist CP-376395 towards the two receptors. From the unbiased MD simulations, we found that in $\mathrm{CRF}_{1} \mathrm{R}$ the oxygen atom on Tyr356 $6^{6.63}$ forms a hydrogen bond with the side chain of His $228^{3.40}$ which allows the formation of a bottleneck consisting of the residues Phe $232^{3.44}$ and Tyr356 ${ }^{6.63}$, while in $\mathrm{CRF}_{2} \mathrm{R}$, the side chain oxygen on Tyr $323^{6.63}$ is hydrogen bonded to the side chain of $G \ln 269^{6.44}$, leading to a lack of such a bottleneck. The existence of the bottleneck in $\mathrm{CRF}_{1} \mathrm{R}$ and its absence in $\mathrm{CRF}_{2} \mathrm{R}$ provide an explanation for the origin of the high selectivity of the antagonist CP-376395 towards $\mathrm{CRF}_{1} \mathrm{R}$. The metadynamics simulations provided an even stronger support for that explanation. The rotameric switch of the side chain of Tyr $356^{6.63}$ results in the breaking down of the bottleneck in $C F_{1} R$ and is a prerequisite for the dissociation of $\mathrm{CP}-376395$ from $\mathrm{CRF}_{1} \mathrm{R}$, but it is not required for the dissociation of CP-376395 from $\mathrm{CRF}_{2} \mathrm{R}$ as indicated by the FES. Thus, our studies provide important structural information in explaining the origin of the high selectivity of CP-376395 towards $\mathrm{CRF}_{1} \mathrm{R}$.

\section{Methods}

Homology modeling of $\mathrm{CRF}_{2} \mathbf{R}$. The crystal structure of $\mathrm{CRF}_{1} \mathrm{R}$ (PDB entry ID: $4 \mathrm{~K} 5 \mathrm{Y})^{1}$ was used as the template for the homology modeling of the structure of $\mathrm{CRF}_{2} \mathrm{R}$. ClustalW2 was used for the sequence alignments and manual adjustments were carried out to guarantee no gaps in the secondary structures ${ }^{27}$. Prime 3.5 was used to build the model of $\mathrm{CRF}_{2} \mathrm{R}^{28,29}$.

Unbiased molecular dynamics simulations. System preparation. Two systems, one containing the crystal structure of $\mathrm{CRF}_{1} \mathrm{R}$ and the other the modeled structure of $\mathrm{CRF}_{2} \mathrm{R}$, were built for the simulations. A POPC (1-palmitoyl-2-oleoyl-sn-glycero-3phosphocholine) bilayer with the surface area of $75 \AA \times 75 \AA$ on the X-Y plane was constructed using $\mathrm{VMD}^{13}$. For each system, the receptor was first embedded into the POPC bilayer using our in-house program according to the orientations provided by the OPM database ${ }^{30}$. The antagonist CP-376395 was placed in the antagonist binding pocket. A box of $75 \times 75 \times 100 \AA^{3}$ with water molecules was then used to solvate the protein. Lipid molecules within $0.85 \AA$ of the heavy atoms on the protein structure and water molecules in the bilayer were removed. Thereafter, sodium and chloride

Table 1 | Systems prepared for the MD simulations

\begin{tabular}{lcccr} 
System ID & Protein & POPC & $\mathrm{Na}$ & $\mathrm{Cl}-$ \\
\hline $\mathrm{A}$ & $\mathrm{CRF}_{1} \mathrm{R}$ & 103 & 51 & 60 \\
$\mathrm{~B}$ & $\mathrm{CRF}_{2} \mathrm{R}$ & 103 & 51 & water \\
\hline
\end{tabular}


ions were added to produce the neutral system of $0.15 \mathrm{M} \mathrm{NaCl}$. The resulting systems are summarized in Table 1.

Simulation details. MD simulations were performed using Gromacs 4.6.5 $5^{31,32}$ with the CHARMM36 parameters for the proteins, lipids, and ions and the TIP3P model for water. Force field parameters for the ligand molecule were generated with the CHARMM General Force Field (version 2b8) interface (version 0.9.7.1 beta) ${ }^{33}$ and were listed in Appendix 1. Three steps were used to equilibrate each system. In the first step, the system was subject to a 50000-step energy minimization with $1000.0 \mathrm{~kJ} /$ $\mathrm{mol} / \mathrm{nm}$ as the force threshold. Then, the system was relaxed by an MD simulation of 100 ps with $1 \mathrm{fs}$ as the time step using the NVT ensemble. In the last step, the system underwent an NPT MD simulation for $1 \mathrm{~ns}$ with the time step of 2 fs for equilibration.

After the equilibration run, each system was simulated for 50 ns using the NPT ensemble with the temperature and pressure set to $310 \mathrm{~K}$ and 1 bar, respectively. The Nose-Hoover thermostat and the Parrinello-Rahman pressure coupling were applied during the simulation. The bonds containing hydrogen atoms were constrained with the LINCS algorithm and a time step of $2 \mathrm{fs}$ was used. The cubic periodic boundary conditions were applied. The cut-offs for the electrostatic and van der Waals interactions were set to $12 \AA$, with the long range electrostatic interactions recovered by the Particle Mesh Ewald summation.

Metadynamics simulations. Theory. Metadynamics ${ }^{34-36}$ has been successfully applied in describing the selectivity of a ligand towards different targets $s^{37}$. We performed metadynamics simulations to detect the residues that are relevant to the selectivity of CP-376395 towards $\mathrm{CRF}_{1} \mathrm{R}$ and $\mathrm{CRF}_{2} \mathrm{R}$. In a metadynamics simulation, an additional history-dependent biased potential $V_{G}(S, t)$ was introduced into the system,

$$
V_{G}(S, t)=\int_{0}^{t} d t^{\prime} \omega \exp \left(-\sum_{i=1}^{d} \frac{\left(S_{i}(R)-S_{i}\left(R\left(t^{\prime}\right)\right)\right)^{2}}{2 \sigma_{i}^{2}}\right)
$$

where $t$ represents time, $S$ represents the collective variables, $\omega$ is the energy rate and $\sigma_{i}$ controls the width of the Gaussian for the $i$ th collective variable. With the evolution of the system, the wells in the FES of the collective variables are filled up with the biased potential $V_{G}$. The underlying free energy $-F(S)$ is assumed to be estimated from the biased potential once all the wells have been filled after a sufficiently long time,

$$
\lim _{\mathrm{t} \rightarrow \infty} V_{G}(S, t) \sim-F(S)
$$

The correctness of the relationship as shown in equation 2 has proven to be empirical by extensive tests under the assumption that the stochastic dynamics in the collective variable space is memoryless in the absence of the bias. Under the assumption, the error in FES construction has proven to be:

$$
\varepsilon \propto \sqrt{\frac{\omega}{D\left(k_{B} T\right)^{-1}}}
$$

Where $\mathrm{D}$ is the intrinsic system diffusion coefficient in the collective variable space, $k_{B}$ is the Boltzmann constant, and $\mathrm{T}$ is the temperature of the system ${ }^{38}$.

In fact, if one is interested in reconstructing the free energy surface from a metadynamics simulation, the simulation should be stopped once the biased potential fulfills the underlying FES in the region of interest. If the simulation does not stop as soon as the system exits from the minima, the biased potential would overfill the minima and push the system to high energy regions with respect to the collective variable space. To solve the problem, a "well-tempered" and "smoothly converging" algorism is introduced by Barducci et al $^{39}$. In the well-tempered metadynamics, the deposition rate for the biased potential decreases by rescaling the Gaussian height (W) over the simulation time

$$
\mathrm{W}=\omega \tau_{G} e^{\frac{V_{c}(S, t)}{k_{B} \Delta T}},
$$

where $V_{G}(S, t)$ is the biased potential at the current position and current time, $\tau_{G}$ is the deposition stride, and $\Delta \mathrm{T}$ is a temperature-like parameter. The underlying free energy is a scaled approximation to the $V_{G}(S, t)$, with

$$
F(S)=-\frac{\Delta T}{T+\Delta T} V_{G}(S, t \rightarrow \infty),
$$

With respect to the standard metadynamics, the biased potential decreases as $1 / \mathrm{t}$ when the simulation proceeds, which allows to smoothly converge to an approximation of $F(S)$

Simulation details. We have carried out 50 ns well-tempered metadynamics simulations for the systems of $\mathrm{CRF}_{1} \mathrm{R}$ and $\mathrm{CRF}_{2} \mathrm{R}$ with the antagonist $\mathrm{CP}-376395$ in their antagonist binding pockets. For each system, the metadynamics simulations were carried out two times, with the last snapshot from the unbiased MD simulation used as the initial structure for the simulations. The metadynamics simulations were carried out using plumed 2.02 implemented in Gromacs 4.6.5. The collective variables were selected based on the unbiased MD simulations. The residues $\mathrm{Phe}^{3.44}$ and $\mathrm{Tyr}^{6.63}$ have been suggested to be working as the bottleneck for the binding of CP-376395 to its binding pocket ${ }^{1}$. We have observed a rotational switch of Tyr323 $3^{6.63}$ in $\mathrm{CRF}_{2} \mathrm{R}$ in the unbiased MD simulation. This rotational switch opens the bottleneck that controls the binding of CP-376395 to the antagonist binding pocket. Thus, the $\chi_{1}$ torsional angle of $\mathrm{Tyr}^{6.63}$ was selected to control the opening and closing of the bottleneck and used as the first collective variable. The nitrogen on the dimethylpyridine group of the antagonist forms a hydrogen bond with the side chain of $A_{s n}^{5.50}$ in $\mathrm{CRF}_{1} \mathrm{R}$ and $\mathrm{CRF}_{2} \mathrm{R}$. This hydrogen bond plays a pivotal role in the binding of CP-376395 to the antagonist binding pockets. In addition, a dissociation pathway along the $\mathrm{Z}$-axis from the antagonist binding pocket to the extracellular side of the receptor $\mathrm{CRF}_{1} \mathrm{R}$ was proposed base on the random acceleration MD simulations ${ }^{16}$. We thus select the Zcomponent of the vector connecting the nitrogen on the dimethylpyridine group of $\mathrm{CP}-376395$ and $\mathrm{C}^{\mathrm{Y}}$ on the $\mathrm{Asn}^{5.50}$ as the second collective variable. For the metadynamics simulations, the biasing potential was added every 250 steps, with the width and height of the Gaussian hills set to 0.05 and $0.3 \mathrm{~kJ} / \mathrm{mol}$, respectively, and $\Delta \mathrm{T}=$ 2700 .

1. Hollenstein, K. et al. Structure of class B GPCR corticotropin-releasing factor receptor 1. Nature 499, 438-443 (2013).

2. Vale, W., Spiess, J., Rivier, C. \& Rivier, J. Characterization of a 41-residue ovine hypothalamic peptide that stimulates secretion of corticotropin and betaendorphin. Science 213, 1394-1397 (1981).

3. Owens, M. J. \& Nemeroff, C. B. Physiology and pharmacology of corticotropinreleasing factor. Pharmacol. Rev. 43, 425-473 (1991).

4. Janssen, D. \& Kozicz, T. Is it really a matter of simple dualism? Corticotropinreleasing factor receptors in body and mental health. Front. Endocrinol. 4, 28 (2013).

5. Refojo, D. et al. Glutamatergic and dopaminergic neurons mediate anxiogenic and anxiolytic effects of CRHR1. Science 333, 1903-1907 (2011).

6. Giardino, W., Mark, G., Stenzel-Poore, M. \& Ryabinin, A. Dissociation of corticotropin-releasing factor receptor subtype involvement in sensitivity to locomotor effects of methamphetamine and cocaine. Psychopharmacology 219 1055-1063 (2012).

7. Fleck, B. A., Hoare, S. R. J., Pick, R. R., Bradbury, M. J. \& Grigoriadis, D. E. Binding Kinetics Redefine the Antagonist Pharmacology of the Corticotropin-Releasing Factor Type 1 Receptor. J. Pharmacol. Exp. Ther. 341, 518-531 (2012).

8. Magnani, F. et al. Electronic Sculpting of Ligand-GPCR Subtype Selectivity: The Case of Angiotensin II. ACS Chem. Biol. 9, 1420-1425 (2014).

9. Chen, Y. L. et al. 2-Aryloxy-4-alkylaminopyridines: Discovery of Novel Corticotropin-Releasing Factor 1 Antagonists. J. Med. Chem. 51, 1385-1392 (2008).

10. Wang, J. \& Verkhivker, G. M. Energy Landscape Theory, Funnels, Specificity, and Optimal Criterion of Biomolecular Binding. Phys. Rev. Lett. 90, 188101 (2003).

11. Wootten, D., Simms, J., Miller, L. J., Christopoulos, A. \& Sexton, P. M. Polar transmembrane interactions drive formation of ligand-specific and signal pathway-biased family B G protein-coupled receptor conformations. Proc. Natl. Acad. Sci. U.S.A. 110, 5211-5216 (2013).

12. Lovell, S. C. et al. Structure validation by $\mathrm{C} \alpha$ geometry: $\varphi, \Psi$ and $C \beta$ deviation. Proteins: Struct. Funct. Bioinf. 50, 437-450 (2003).

13. Rosenbaum, D. M., Rasmussen, S. G. F. \& Kobilka, B. K. The structure and function of G-protein-coupled receptors. Nature 459, 356-363 (2009).

14. Venkatakrishnan, A. J. et al. Molecular signatures of G-protein-coupled receptors. Nature 494, 185-194 (2013).

15. Hjorth, S. A., Orskov, C. \& Schwartz, T. W. Constitutive activity of glucagon receptor mutants. Mol. Endocrinol. 12, 78-86 (1998).

16. Bai, Q., Shi, D., Zhang, Y., Liu, H. \& Yao, X. Exploration of the antagonist CP376395 escape pathway for the corticotropin-releasing factor receptor 1 by random acceleration molecular dynamics simulations. Mol. Biosyst. 10 1958-1967 (2014).

17. Dror, R. O. et al. Activation mechanism of the beta2-adrenergic receptor. Proc Natl. Acad. Sci. U. S. A. 108, 18684-18689 (2011).

18. Dror, R. O. et al. Identification of two distinct inactive conformations of the beta2 adrenergic receptor reconciles structural and biochemical observations. Proc. Natl. Acad. Sci. U. S. A. 106, 4689-4694 (2009).

19. Lebon, G., Warne, T. \& Tate, C. G. Agonist-bound structures of G protein-coupled receptors. Curr. Opin. Struct. Biol. 22, 482-490 (2012).

20. Moukhametzianov, R. et al. Two distinct conformations of helix 6 observed in antagonist-bound structures of a $\beta 1$-adrenergic receptor. Proc. Natl. Acad. Sci. U.S.A. 108, 8228-8232 (2011).

21. Bhattacharya, S., Hall, S. E., Li, H. \& Vaidehi, N. Ligand-Stabilized Conformational States of Human $\beta(2)$ Adrenergic Receptor: Insight into GProtein-Coupled Receptor Activation. Biophys. J. 94, 2027-2042 (2008).

22. Liaw, C. W., Grigoriadis, D. E., Lorang, M. T., De Souza, E. B. \& Maki, R. A. Localization of Agonist- and Antagonist-Binding Domains of Human Corticotropin-Releasing Factor Receptors. Mol. Endocrinol. 11, 2048-2053 (1997).

23. Pan, A. C., Borhani, D. W., Dror, R. O. \& Shaw, D. E. Molecular determinants of drug-receptor binding kinetics. Drug Discov. Today 18, 667-673 (2013).

24. Copeland, R. A., Pompliano, D. L. \& Meek, T. D. Drug-target residence time and its implications for lead optimization. Nat. Rev. Drug Discov. 5, 730-739 (2006).

25. Wang, C. et al. Structural basis for Smoothened receptor modulation and chemoresistance to anticancer drugs. Nat. Commun. 5, 4355 (2014). 
26. Wang, C. et al. Structure of the human smoothened receptor bound to an antitumour agent. Nature 497, 338-343 (2013).

27. Thompson, J. D., Higgins, D. G. \& Gibson, T. J. CLUSTAL W: improving the sensitivity of progressive multiple sequence alignment through sequence weighting, position-specific gap penalties and weight matrix choice. Nucleic. Acids. Res. 22, 4673-4680 (1994).

28. Jacobson, M. P. et al. A hierarchical approach to all-atom protein loop prediction. Proteins: Struct. Funct. Bioinf. 55, 351-367 (2004).

29. Humphrey, W., Dalke, A. \& Schulten, K. VMD: visual molecular dynamics. J. Mol. Graph. 14, 33-38 (1996).

30. Lomize, M. A., Lomize, A. L., Pogozheva, I. D. \& Mosberg, H. I. OPM: orientations of proteins in membranes database. Bioinformatics 22, 623-625 (2006).

31. Van Der Spoel, D. et al. GROMACS: fast, flexible, and free. J. Comput. Chem. 26, 1701-1718 (2005)

32. Hess, B., Kutzner, C., van der Spoel, D. \& Lindahl, E. GROMACS 4: Algorithms for Highly Efficient, Load-Balanced, and Scalable Molecular Simulation. J Chem. Theory. Comput. 4, 435-447 (2008).

33. Vanommeslaeghe, K. et al. CHARMM general force field: A force field for druglike molecules compatible with the CHARMM all-atom additive biological force fields. J. Comput. Chem. 31, 671-690 (2010).

34. Laio, A. \& Parrinello, M. Escaping free-energy minima. Proc. Natl. Acad. Sci. U.S.A. 99, 12562-12566 (2002).

35. Bonomi, M. et al. PLUMED: A portable plugin for free-energy calculations with molecular dynamics. Comput. Phys. Commun. 180, 1961-1972 (2009).

36. Tribello, G. A., Bonomi, M., Branduardi, D., Camilloni, C. \& Bussi, G. PLUMED 2 New feathers for an old bird. Comput. Phys. Commun. 185, 604-613 (2014).

37. Limongelli, V. et al. Molecular basis of cyclooxygenase enzymes (COXs) selective inhibition. Proc. Natl. Acad. Sci. U.S.A. 107, 5411-5416 (2010).

38. Barducci, A., Bonomi, M. \& Parrinello, M. Metadynamics. Wiley Interdiscip. Rev. Comput. Mol. Sci. 1, 826-843 (2011).
39. Barducci, A., Bussi, G. \& Parrinello, M. Well-Tempered Metadynamics: A Smoothly Converging and Tunable Free-Energy Method. Phys. Rev. Lett. 100 (2008).

\section{Acknowledgments}

This work was supported by the grants from the Swedish National Infrastructure for Computing (SNIC), SNIC2013-26-31 and SNIC2013-1-236. X.S. acknowledges the China Scholarship Council for financial support.

\section{Author contributions}

X.S. and X.W. carried out the molecular dynamics simulations. X.S. and Y.T. designed the study and analyzed the data. Y.T. was responsible for the project. X.S., J.C., Y.T., H.A. and Y.T. contributed to writing and commenting on the manuscript.

\section{Additional information}

Supplementary information accompanies this paper at http://www.nature.com/ scientificreports

Competing financial interests: The authors declare no competing financial interests.

How to cite this article: Sun, X. et al. Residues remote from the binding pocket control the antagonist selectivity towards the corticotropin-releasing factor receptor-1. Sci. Rep. 5, 8066; DOI:10.1038/srep08066 (2015).

This work is licensed under a Creative Commons Attribution-NonCommercialNoDerivs 4.0 International License. The images or other third party material in this article are included in the article's Creative Commons license, unless indicated otherwise in the credit line; if the material is not included under the Creative Commons license, users will need to obtain permission from the license holder in order to reproduce the material. To view a copy of this license, visit http:// creativecommons.org/licenses/by-nc-nd/4.0/ 